\title{
Onset Time of Solar Cycle 23 GLEs
}

\author{
Y. H. Tang, $\dagger$ C. Li and Y. Dai \\ Department of Nanjing University, Nanjing 210093, China \\ email: yhtang@nju.edu.cn
}

\begin{abstract}
Based on the neutron monitor counting rate profiles, soft X-ray emission from GOES, Yohkoh/HXT, RHESSI/HXT, ACE/SIS, and SOHO/LASCO CME catalog, the relationship between the onset time of solar cycle 23 Ground Level Enhancements(GLEs) and solar activity are analyzed. It was found that the correlation between the onset time of GLEs and flare maximum is better than that of the onset time of metric type II radio burst and CME. Average value of the ratio of $\mathrm{Fe} / \mathrm{O}$ is about 2.65 times of coronal value in the range $30-40 \mathrm{MeV} / \mathrm{n}$ for all events. If considering the parent flare sites, the situation of proton acceleration in flares, and transport effect from flare sites (if it located in poorly-connected region) to well-connected region, several GLEs onset time are roughly at the peak time of the X-ray.
\end{abstract}

\section{Data analysis}

In order to discuss the relationship between the onset time of solar cycle 23 GLEs and solar activity, the relevant observations are given in Table 1 . Where $T_{G L E}, T_{\text {Max }}, T_{\text {peak }}$, $T_{I I m}, T_{C M E}$ denote the onset of GLEs, soft X-ray maximum, the main peak time of hard $\mathrm{X}$-ray ( $\gamma$-ray), the onset of metric type II radio bursts, and CME onset respectively. The ratio of Fe/O in last column are taken from Tylka et al (2005 Table 1).

Since many authors showed the nuclear line emission during the flares was first detected from the peak of hard X-ray or $\gamma$-ray through the decay phase. Therefore, we set $\triangle T_{1}=$ $T_{\text {event }}-T_{\text {max }}$, and $\triangle T_{2}=T_{\text {event }}-T_{\text {peak }}$, which are defined as the time interval from the soft X-ray maximum and main peak of hard X-ray or $\gamma$-ray emission to the onset of GLE event respectively; $\triangle T_{3}$ and $\triangle T_{4}$ are defined as the time interval from the onset of

$\dagger$ Present address: Department of Astronomy, Nanjing University, Nanjing, China

Table 1. GLE events of solar cycle 23 and their associated characteristic

\begin{tabular}{l|c|c|c|c|c|c|c|c|c}
\hline No. & Date & $\begin{array}{c}T_{G L E} \\
\text { (UT) }\end{array}$ & flare & $\begin{array}{c}T_{\text {Max }} \\
\text { (UT) }\end{array}$ & $\begin{array}{c}T_{\text {peak }} \\
(\mathrm{UT})\end{array}$ & $\begin{array}{c}T_{\text {IIm }} \\
(\mathrm{UT})\end{array}$ & $\begin{array}{c}T_{C M E} \\
(\mathrm{UT})\end{array}$ & connection & Fe/O/0.134 \\
\hline 55 & $06 / 11 / 97$ & $12: 07$ & S18W63 & $11: 55$ & $(11: 58)$ & $11: 53$ & $11: 39$ & well & 5.78 \\
56 & $02 / 05 / 98$ & $13: 53$ & S15W15 & $13: 42$ & $11: 38$ & $13: 41$ & $13: 32$ & poorly & 4.93 \\
57 & $06 / 05 / 98$ & $08: 22$ & S11W65 & $08: 09$ & $08: 05$ & $08: 03$ & $07: 55$ & well & 3.99 \\
58 & $24 / 08 / 98$ & $22: 47$ & N35E09 & $22: 04$ & No & $22: 02$ & No & poorly & 0.84 \\
59 & $14 / 07 / 00$ & $10: 30$ & N22W07 & $10: 24$ & $(10: 27)$ & $10: 28$ & $10: 25$ & poorly & 0.57 \\
60 & $15 / 04 / 01$ & $13: 57$ & S20W85 & $13: 50$ & $13: 49$ & $13: 47$ & $13: 35$ & well & 4.78 \\
61 & $18 / 04 / 01$ & $02: 32$ & S23W120 & $02: 14$ & $02: 15$ & $02: 17$ & $02: 11$ & well & 2.95 \\
62 & $04 / 11 / 01$ & $16: 57$ & N06W18 & $16: 20$ & $16: 17$ & $16: 10$ & $16: 13$ & poorly & 0.31 \\
63 & $26 / 12 / 01$ & $05: 27$ & N08W54 & $05: 40$ & No & $05: 12$ & $05: 06$ & well & 4.18 \\
64 & $24 / 08 / 02$ & $01: 15$ & S02W81 & $01: 12$ & No & $01: 01$ & $00: 59$ & well & 4.61 \\
65 & $28 / 10 / 03$ & $11: 19$ & S20E02 & $11: 10$ & $(11: 06)$ & $11: 02$ & $11: 07$ & poorly & 0.068 \\
66 & $29 / 10 / 03$ & $21: 27$ & S19W09 & $20: 49$ & $20: 40$ & $20: 41$ & $20: 43$ & poorly & 0.83 \\
67 & $02 / 11 / 03$ & $17: 27$ & S18W59 & $17: 25$ & No & $17: 14$ & $17: 19$ & well & 0.57 \\
68 & $20 / 01 / 05$ & $06: 48$ & N14W61 & $07: 01$ & $(06: 46)$ & $06: 44$ & $06: 33$ & well & \\
\hline
\end{tabular}


Table 2. Time interval between the onset of GLEs and solar activity

\begin{tabular}{c|c|c|c|c|c|c|c|c|c|c|c|c|c|c|c|c|c|c}
\hline GLE No & 55 & 56 & 57 & 58 & 59 & 60 & 61 & 62 & 63 & 64 & 65 & 66 & 67 & 68 \\
\hline$\triangle T_{1}($ min $)$ & 12 & 11 & 13 & 35 & 6 & 7 & 18 & 37 & -13 & 3 & 9 & 38 & 2 & -13 \\
$\triangle T_{2}($ min $)$ & 9 & 15 & 17 & No & 3 & 8 & 17 & 40 & No & No & 13 & 47 & No & 2 \\
$\triangle T_{3}($ min $)$ & 14 & 12 & 19 & 45 & 2 & 10 & 15 & 47 & 15 & 14 & 17 & 46 & 13 & 4 \\
$\triangle T_{4}($ min $)$ & 28 & 21 & 27 & No & 5 & 22 & 21 & 44 & 21 & 16 & 12 & 44 & 8 & 15 \\
\hline
\end{tabular}

Table 3. Correlation among onset of GLE, flare Max., type II metric radio burst, and CME onset

\begin{tabular}{c|c|c|c|c}
\hline$\triangle T$ & $\frac{N_{\triangle T_{1}}}{N_{\text {total }}}$ & $\frac{N_{\triangle T_{2}}}{N_{\text {total }}}$ & $\frac{N_{\triangle T_{3}}}{N_{\text {total }}}$ & $\frac{N_{\Delta T_{4}}}{N_{\text {total }}}$ \\
\hline \multirow{210\mathrm{min}}{*}{} & 0.357 & 0.40 & 0.214 & 0.154 \\
$\leqslant 20 \mathrm{~min}$ & 0.429 & 0.40 & 0.572 & 0.23 \\
$>20 \mathrm{~min}$ & 0.214 & 0.20 & 0.214 & 0.616 \\
\hline
\end{tabular}

metric type II and CME onset to the onset of GLE event respectively. The results are showed in Table 2.

Because of the confinement by spiral IMF lines, only GLEs from source regions > $W 30^{\circ}$ in longitude can directly propagate to observers near the Earth, unlike those of well-connected events which have source regions $>W 30^{\circ}$, GLEs in poorly connected events first are to be transported to the Earth-connected IMF lines and then could be observed. We re-divide the events in Table 1 into two categories: well-connected (i.e., events 55, 57, 60, 61, 63, 64, 67, 68)and poorly connected (i.e., events 56, 58, 59, 62, $65,66)$, and examine the action of transport effect. It was found that the average time interval $\triangle T_{1}$, for well-connected events, it is about 10 minutes, but for poorly-connected events is 23 minutes.

The correlation between the onset time of GLEs, and solar activity (soft X-ray maximum, hard X-ray or $\gamma$-ray main peak, onset of metric type II radio burst, and CME onset) are listed in Table 3. From Table 3, we found that the correlation between the onset of GLEs and hard X-ray or $\gamma$-ray main peak, as well as soft X-ray maximum are better than that of metric type II radio bursts and CMEs onset.

\section{Summary}

From the above analysis, the conclusions can be briefly summarized as follows: (1). From Table 3, it was found that the correlation between the onset of GLEs and hard $\mathrm{X}$-ray or $\gamma$-ray main peak, as well as soft X-ray maximum are better than that of metric type II radio bursts and CMEs onset. (2). Average value of the ratio of $\mathrm{Fe} / \mathrm{O} / 0.134$ $(30-40 \mathrm{MeV} / \mathrm{n})$ is about 2.65 for all events; for well-connected events, average value is about 3.84, for poorly-connected events it is about 1.26. (3). Transport effect from flare sites (if it located in poorly-connected region) to well-connected region is obvious, for well-connected events, the average time interval $\triangle T_{1}$ is about 10 minutes, but for poorly-connected events is about 23 minutes.

\section{References}

Tylka, A. J., Cohen, C. M., Dietrich, W. F. et al., 2005, ApJ, 625, 474-495

Li, C., Tang, Y. H., Dai, Y. et al., 2006, ApJ, submitted. 